\title{
Secondary Prevention of Systemic Arterial and Recurrent Venous Thromboembolic Events, Through Extended Time Anticoagulation with Apixaban, in an Elderly Patient with Multiple Comorbidities, Clinical Case
}

Aurelian Anghelescu1,2

\section{Abstract}

The clinical case illustrates the daily practice with an individualized, adjusted thromboprophylactic dose of apixaban (2.5 mg twice-daily, follow-up for 20 months), mandatory indicated as a continuous therapy for secondary prevention of systemic arterial embolism and recurrent venous thromboembolic events, in an elderly obese patient, associating multiple pathological conditions (chronically anticoagulated for non valvular atrial fibrillation and stroke sequelae, multiple metabolic comorbidities such as type 2 diabetes mellitus, moderate renal insufficiency, hyperuricemia), who submitted a severe "unprovoked" phlegmasia alba dolens in the sound (left) lower limb, a decade after a (most possible) iatrogenic deep vein thrombosis at the paralyzed (right) limb.

Thromboprophylaxis for an extended time, without any medical complications (neither systemic or venous thrombotic events, nor hemorrhagic ones) confirmed the product's efficacy and safety profile. Physiopathological considerations and essential pharmacological considerations were discussed, focused on the individualized safe therapeutic approach.

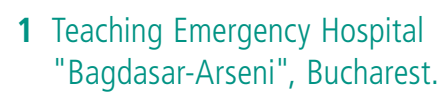

2 "Carol Davila" University of Medicine and Pharmacy, Bucharest.

\section{Contact information:}

\section{Aurelian Anghelescu.}

Neurorehabilitation Clinic, Teaching Emergency Hospital "Bagdasar-Arseni".

Address: Berceni Av., No.12, postal code: 041915, 4th Sector, Bucharest, Romania. Tel: +4021/ $3343025(6,7) / 1003$.

Mobil phone: +40720807607 .

” aurelian_anghelescu@yahoo.co.uk

Keywords

Thromboprophylaxis; Deep Vein Thrombosis; Vitamin $\mathrm{K}$ Antagonist (Acenocumarol); Novel/Target-Specific/Direct Oral Anticoagulant (NOACs, TSOAs, DOACs); stroke sequelae.

\section{Introduction}

Contemporary registers for randomized evaluation of long-term anticoagulant therapy indicated for thromboprophylaxis in chronic atrial fibrillation (GARFIELD 60,000 patients in 34 countries, RE-LY AF 15,400 patients in 46 countries) revealed that in North America only $65.7 \%$ of 
patients with atrial fibrillation (AF) at risk for thromboembolic events are treated with oral anticoagulants [1].

Efficient secondary cardioembolic stroke prophylaxis with oral anticoagulants has a large medical and socioeconomic variation, between developed and poor countries. The mean time in the therapeutic range (TTR) in Western Europe was found to be $62.4 \%$, high in North America $50.9 \%$, but tiny in India, China, Southeast Asia and Africa (TTR between 32\%-40\%) [1].

Amid an imperfect anticoagulation may occur systemic or venous thrombotic events, with or without evident trigger or risk factors.

Deep vein thrombosis (DVT) and pulmonary embolism (PE), collectively denominated venous thromboembolism (VTE), represent a common, severe clinical condition, associated to significant morbidity and mortality, but preventable. Despite adequate curative and prophylactic treatment, many patients (approximately 10\%-30\%) with symptomatic DVT and/or PE will experience a recurrent thrombotic event within subsequent years [2].

\section{Case Presentation}

A 65 years-old man, non-smoker, with a past medical history significant for metabolic syndrome (obesity IMC= 31.8; 92 kg, $170 \mathrm{~cm}$, type 2 diabetes equilibrated with diet, dyslipidemia and hypertension) was diagnosed in 2004 (at 55 years-old) with non valvular atrial fibrillation (NVAF) complicated with cardio-embolic stroke in the left middle cerebral artery territory.

In 2004 was admitted in our Rehabilitation Clinic, in post-acute stage after stroke, with a flaccid right hemiplegia, global aphasia, and an acute proximal deep vein thrombosis (DVT) at the right lower limb, (most probably) iatrogenic post incorrectly administration of infusions in the superficial veins of the ankle. The acute venous episode was adequately treated with a low-molecular-weight heparin (LMWH). He was discharged on medication for blood pressure control and antiarithmics, neurotrophics, statin, and anticoagulation with a vitamin $\mathrm{K}$ antagonist (VKA, acenocumarol/sintrom), successfully administered for both prophylactic targets: secondary prevention of cardio-embolic stroke or non-(central nervous system) CNS embolism, and DVT recurrence.

He lived in the family, dependent for most of the basic ADL items, with disabling sequelae: right spastic hemiparesis (with typical arterial topography) and mixed aphasia (mainly expressive). He was able to walk for short distances, assisted by his wife, and had no residual postphlebitis syndrome.

During the entire decade 2004-2014 he was chronically anticoagulated with the above-mentioned VKA. He did not receive any factor incriminated in possible INR modification (occasionally antibiotic therapy, but neither amiodarone, herbal, aspirin, nor alcohol consumption). The retrospective estimation of how long the patient was efficiently anticoagulated (therapeutic time in range, TTR\%) is depicted in Figure 1. From a total number of 43 tests, 33 were within range (TTR 76.7\%)

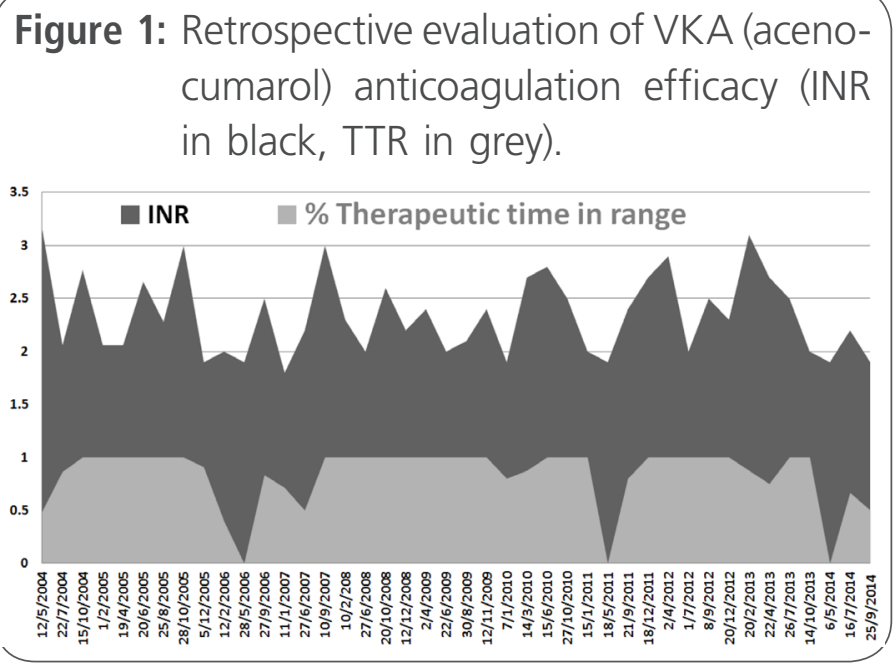

In time, the patient gradually developed a moderate chronic kidney disease (CKD); in only a few occasions the creatinine clearance $(\mathrm{CrCl})$ had a tiny decrease under the upper edge of $30 \mathrm{ml} /$ min $/ 1.73 \mathrm{~m}^{2}$ (considered borderline for a severe 
Figure 2: Retrospective evaluation of the chronic kidney disease (blood creatinine and creatinine clearance)

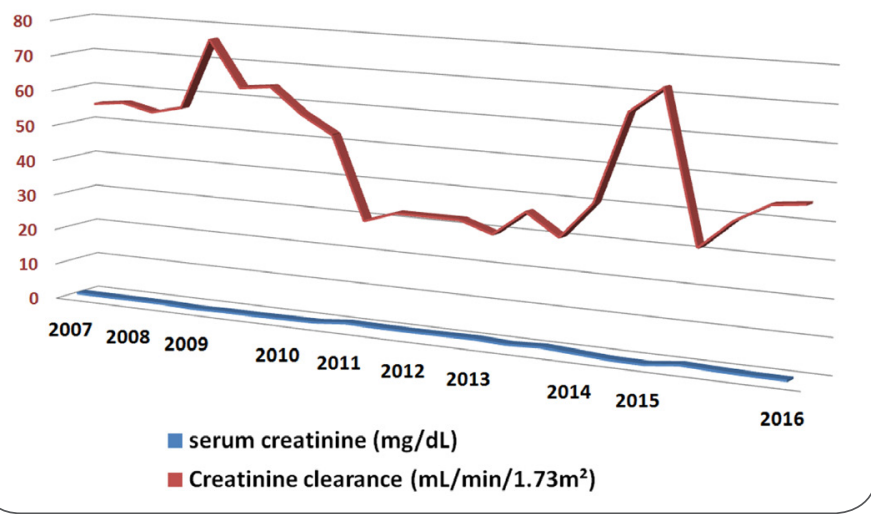

Figure 3: Left DVT phlegmasia alba dolens (2014) with good subsequent outcomes. The contrasting clinical difference between the thickness of the lower limbs is due to the amyotrophic aspect of the right lower limb and the residual post phlebitis syndrome on the left $(2015,2016)$. [Written informed consent was obtained from the patient for publication, as well as our hospital's Bioethics Commission approval].
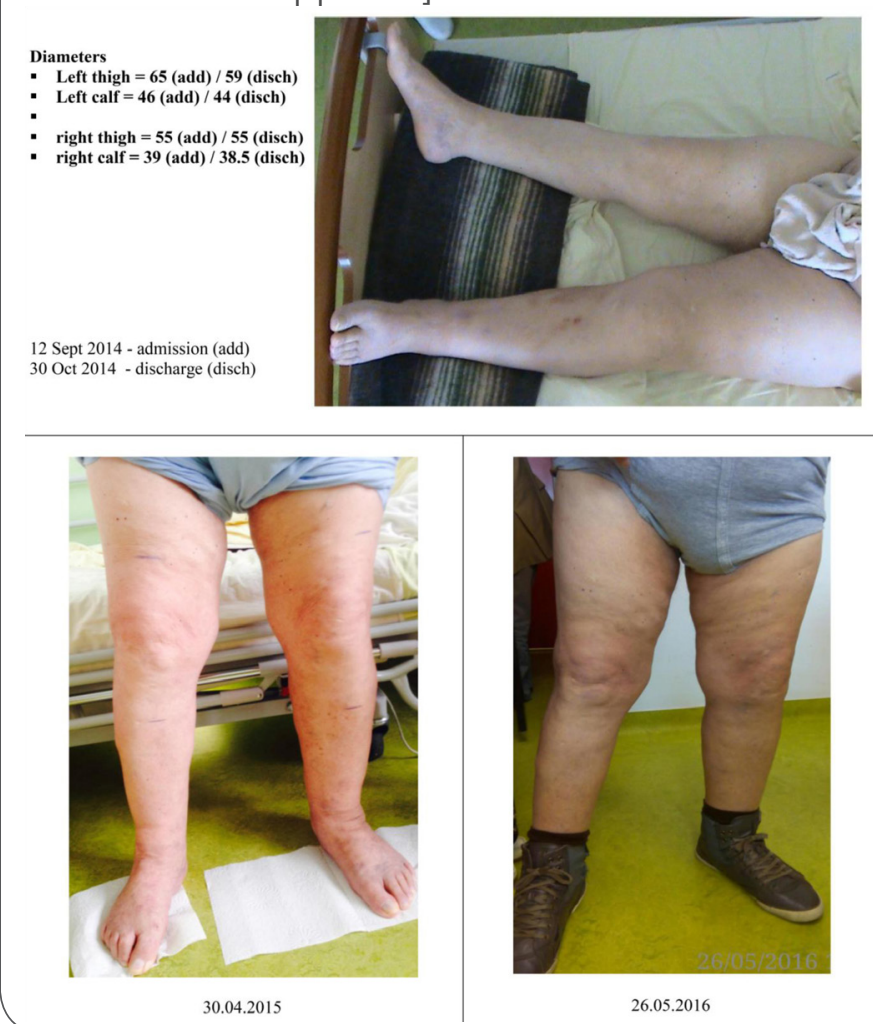

CKD). The retrospective evolution of the blood creatinine values and creatinine clearance is represented in Figure 2.

The "strange" graphic aspect of $\mathrm{CrCl}$ is due to the fact that the patient addressed 4 different private laboratories during 2008-2010, 2011, 2012-2013, 2014-2016; this "perplexed" element was not noticed during isolated consultations.

Hyperuricemia (occurred since 2013, treated with allopurinol $100 \mathrm{mg}$ QD), completed the clinical picture of the metabolic syndrome.

In September 2014 the patient experienced a respiratory viral infection, occurred amid an episodic inefficient anticoagulation treatment with acenocumarol (INR 1.9); in a few days he developed an acute superficial-vein phlebitis of the healthy (left) shank, misdiagnosed and neglected (treated at home with topic NSAIDs and heparin ointments).

Evolution was rapidly progressive to an extensive ascendant proximal left DVT (Figure 3). He was admitted in our Rehabilitation Clinic presenting the classical signs of phlegmasia alba dolens, with pain at rest and mobilization (in standing and walking), and an "aggressive" trend for bilaterally extension to the common femoral and iliac veins (referred as iliofemoral DVT). Local examination revealed an obvious trajectory of the safena magna vein (visible, palpable and tender).

Significant laboratory coagulation tests (at admission, in 2014) revealed a therapeutically ineffective prothrombin time (PT/ INR 15.5s/1.9) and elevated fibrinogen $765 \mathrm{mg} / \mathrm{dl}$. Post therapeutically values of fibrinogen decreased at $648 \mathrm{mg} / \mathrm{dL}$, respectively at $345 \mathrm{mg} / \mathrm{dL}$.

D-dimer assay had also a favourable outcome (from $1 \mathrm{mcg} / \mathrm{mL}$ at admission, decreased to 0.7 $\mathrm{mcg} / \mathrm{mL}$ post therapeutically, and normalised at discharge to $0.5 \mathrm{mcg} / \mathrm{mL}$ ), in parallel with seriated ultrasound (venous duplex Doppler) examinations, who confirmed the recanalization of the deep veins.

MR Angiography of the iliofemoral veins was not available (from objective technical reasons). 
Routine X-ray of the chest pointed out left ventricle hypertrophy. Cerebral CT confirmed the ischemic stroke sequelae. ECG detected ST-T wave abnormalities (ischemic heart disease) and AF.

Based on the clinical picture, history and absence of evident thrombotic risk factors, the left iliofemoral DVT episode was considered as an "idiopathic"/"unprovoked" thrombotic event.

\section{Discussion}

The clinical case has some particularities to consider:

Occurrence of two DVT severe episodes, at each separate limbs, temporo-spatially distributed at 10 years interval, the first (most probably) iatrogenic, the last (apparently) "unprovoked", with a rapidly progressive ascendant evolution, localized at the non-paralyzed lower limb, in a patient previously (but non constantly efficient) anticoagulated with VKA

Uncommon presentation (at admission) as an acute massive venous thrombosis, phlegmasia alba dolens

The etiologic diagnosis has two major "weak points": one is represented by the missing information concerning a "not so uncommon cause, of a common condition", the May-Thurner syndrome, also called iliocaval compression syndrome [3] (resulting from the arterial conflict upon the left common iliac vein, inflicted by the pulsatile compression of the left internal iliac artery), that should be considered in patients with proximal DVT, in absence of identifiable triggers. An invasive venography was not performed and considered unnecessary (due to safety reasons, lack of the technical device in the hospital, and the subsequent good clinical evolution).

The other limitation consists in absence of previous information regarding a possible hypercoagulable disorder, responsible for most cases of spontaneous DVT. Status of hereditary or acquired hypercoagulability was not investigated, because its specific biological markers (antithrombin, protein C, or protein $\mathrm{S}$ deficiencies) were already altered by previous administration of VKA

The respiratory viral infection (recently preceding the thrombotic episode) was incriminated as a possible trigger factor for the iliofemoral DVT in this particular case, probably liked to a transiently elevated level of antiphospholipid antibodies, usually induced by inflammation or infections. In an epidemiological study, Clayton et al, emphasized a strong association between recent respiratory infections and VTE, noticed an increased risk for DVT in the month following infection and for PE in 3 months following infection, both persisting up to a year [4].

Hyperuricemia, considered an independent risk factor for cardiovascular disease in middle-aged and elderly, was out of discussion, due to the fact that urate-lowering therapy (allopurinol) co-administered with acenocoumarol may increase the anticoagulant effect of the VKA.

Clinical prediction rules, biological parameters and ultrasound imagery were used to tailor therapy to the DVT severity. Anticoagulation options for the acute DVT episode included unfractionated heparin, low molecular weight heparin, fondaparinux and the direct oral anticoagulants (DOACs). It was preferred LMWH (Nadroparine/ Fraxiparine 7,600 anti-factor Xa, subcutaneously, twice-daily SQ BID), due to its advantages over unfractionated heparin (predictability, dose-dependent plasma levels, a long half-life permitting once-/or twice-daily administration, without laboratory monitoring, less bleeding risk). The severity of the case imposed a double dose of LMWH than the usually recommended (85 anti-factor Xa IU/Kg) for 20 days, followed by apixaban $2.5 \mathrm{mg}$ BID.

Favourable outcomes were reflected by the clinical evolution of the lower limbs diameters: left thigh $=65 \mathrm{~cm}$ (admission)/55.5 (discharge); left shank = $46 \mathrm{~cm}$ (admission)/41.5 (discharge); the right (paralyzed) thigh $=55 \mathrm{~cm}$ (admission)/52 (discharge), right shank $=39 \mathrm{~cm}$ (admission)/37.5 (discharge). 
Improvement of the biomarkers (D-dimer progressive normalization from $1 \mathrm{mcg} / \mathrm{mL}$ at admission to 0.7 and $0.5 \mathrm{mcg} / \mathrm{mL}$ ), in conjunction with ultrasound serial examinations (confirming the natural remodeling process of the thrombus and the venous recanalization), were the paraclinical decision milestones, indicating the moment to reconsider the anticoagulant for a "long cruise" therapy and the shift to DOAC (apixaban). The FDA recommended dosage to prevent recurrence (2.5 mg PO BID) was fit to the patient's moderate renal impairment. The good clinical outcomes and the quite stable parameters of the serum creatinine, permitted a safely, extended anticoagulation with apixaban, during the last 20 months (Oct 2014-May 2016).

Extended duration of anticoagulation (20 months forwards) targeted both reduction of the risk for stroke and systemic embolism due to NVAF, and prophylaxis of a possible recurrent VTE.

CHEST antithrombotic therapy guidelines for venous thromboembolism (VTE) recommends [5]:

In patients with DVT of the leg or PE and no cancer, a long-term (3 months) anticoagulant therapy with dabigatran, rivaroxaban, apixaban or edoxaban, over vitamin K antagonist (VKA) therapy (Grade $2 \mathrm{~B}$ )

In patients with DVT of the leg or PE who receive extended therapy, no need to change the choice of anticoagulant after the first 3 months (Grade 2C)

Phase III studies indicated that dabigatran, rivaroxaban, apixaban or edoxaban ["novel" NOACs/"direct" DOACs/"target-specific" TSOAs - all the names, being "La même Jeannette, mais autrement coiffée"] are a more convenient alternative with practical advantages, as effective as the conventional therapy with LMWH and vitamin $\mathrm{K}$ antagonists (warfarin, acenocumarol) for the acute VTE [6-10] and for extended prophylaxis.

This class of new discovered oral anticoagulants brings consistent efficacy (reduced recurrent VTE or VTE-related death compared to placebo) [11], relative safety (significantly reducing the risks of major bleeding) $[6,12]$ and convenience, compared with antivitamin K (acenocumrol or warfarin) [13].

From all the selective oral factor Xa inhibitors, apixaban seems to be associated with the lowest risk for bleeding than the other DOAC's in extended treatment of venous thromboembolism [10] and efficiency in both therapeutic and prophylactic doses [14].

Currently, apixaban is approved for secondary prophylaxis of stroke and systemic embolism in patients with NVAF, for the treatment of acute clinical form of DVT/PE (VTE), respectively risk reduction of recurrent VTE following the initial therapy. These last pharmacological targets were extended by FDA in autumn 2014, so the affiliated term "novel" appears ... quite obsolete.

Due to its favorable pharmacokinetic properties (simple BID administration in fixed doses, low interand/or intra-subject variability, multiple elimination pathways, not exclusively limited on either renal or hepatic metabolic mechanisms, not requiring laboratory monitoring) all important aspects in boosting compliance $[15,16]$ both the patient and his wife had a good psychological adherence and discipline to therapy.

The patient had a moderate risk for major bleedings (rated 2 on the HAS-BLED scale) [17]. From a comprehensive list of 14 predisposing factors for bleeding (increasing age, previous bleeding, cancer, renal or liver failure, previous stroke, diabetes, anemia, thrombocytopenia, antiplatelet therapy, poor anticoagulant control, recent surgery, alcohol abuse, frequent falls), only the previous stroke and T2DM could be noticed [18]. The new ABC (age, biomarkers, clinical history) biomarker-based risk score for major bleeding was not available for follow-up, although it seems to have a better discrimination and utility than the HAS-BLED score [19].

The clinical case underlies the importance of an individualized therapeutic approach (after careful assessment of benefits against risks) and confirms the product's efficacy and safety profile, described in 
seven Phase III trials, cumulating more than 21,000 patients $(4,000$ patients in treatment studies, more than 5,000 subjects in prophylaxis studies, with an average total exposure up to 221 days, and respectively more than 11,000 cases with NVAF, treated with apixaban up to 1.7 years) [20]. Careful followup and individualized dose of apixaban reduced simultaneously the risk for both stroke and nonCNS embolism, respectively the risk for intracranial hemorrhage and/or fatal bleeding.

\section{Conclusions}

The clinical case illustrated the daily practice with an individualized, adjusted thromboprophylactic dose of apixaban (2.5 mg BID), mandatory indicated as a continuous therapy for prevention of systemic arterial embolism and recurrent venous thromboembolic events, in an elderly obese patient, associating multiple chronic conditions (NVAF, stroke sequelae, multiple metabolic comorbidities such as T2DM, hyperuricemia, moderate renal insufficiency), and confirmed the product's efficacy and safety profile in extended time therapeutically approach (followedup for 20 months).

\section{Consent}

Written informed consent was obtained from the patient for photos and publication, as well as our hospital's Bioethics Commission approval.

\section{Statement of Human Rights}

The paper respects human rights, and was realized in accordance with the Helsinki Declaration.

\section{Disclosure}

The author reports no conflicts of interest and did not request or receive any form of financial support for this article.

\section{References}

1. Oldgren J, Healey JS, Ezekowitz M, et al. Variations in etiology and management of atrial fibrillation in a prospective registry of 15,400 emergency department patients in 46 countries: The RE-LY AF Registry. Circulation 2014; 15; 129(15):1568-76

2. Gómez-Outes A, Terleira-Fernández Al, Lecumberri R, SuárezGea ML, Vargas-Castrillón E., Direct oral anticoagulants in the treatment of acute venous thromboembolism: a systematic review and meta-analysis. Thromb Res. 2014; 134(4):774-82

3. Jenkins JS, Michael P, Deep Venous Thrombosis: An Interventionalist's Approach, Ochsner J. 2014; 14(4): 633-640

4. Clayton TC, Gaskin M, Meade TW. Recent respiratory infection and risk of venous thromboembolism: case-control study through a general practice database. Int J Epidemiol. 2011; 40(3):819-27

5. Kearon $C$, Akl E, Ornelas J, Blaivas $A$, Jimenez $D$, Bounameaux $H$, et al., Antithrombotic therapy for VTE Disease: Chest Guideline and Expert Panel Report, Chest 2016; 149(2):315-352.

6. Streiff MB, Agnelli G, Connors JM, Crowther M, Eichinger S, Lopes R, McBane RD, Moll S, Ansell J. Guidance for the treatment of deep vein thrombosis and pulmonary embolism. J Thromb Thrombolysis 2016; 41(1):32-67

7. van der Hulle T, Kooiman J, den Exter PL, Dekkers OM, Klok FA, Huisman MV, Effectiveness and safety of novel oral anticoagulants as compared with vitamin $\mathrm{K}$ antagonists in the treatment of acute symptomatic venous thromboembolism: a systematic review and meta-analysis, J Thromb Haemost. 2014; 12(3):320-8

8. Gómez-Outes A, Terleira-Fernández Al, Lecumberri R, SuárezGea ML, Vargas-Castrillón E., Direct oral anticoagulants in the treatment of acute venous thromboembolism: a systematic review and meta-analysis. Thromb Res. 2014; 134(4):774-82

9. Rollins BM, Silva MA, Donovan JL, Kanaan AO., Evaluation of Oral Anticoagulants for the Extended Treatment of Venous Thromboembolism Using a Mixed-Treatment Comparison, Meta-Analytic Approach. Clin Ther 2014; 1; 36(10):1454-64

10. Mantha S, Ansell J., Indirect comparison of dabigatran, rivaroxaban, apixaban and edoxaban for the treatment of acute venous thromboembolism., J Thromb Thrombolysis 2015; 39, 2, 155-165

11. Sardar P, Chatterjee S, Mukherjee D., Efficacy and safety of new oral anticoagulants for extended treatment of venous thromboembolism: systematic review and meta-analyses of randomized controlled trials. Drugs. 2013; 73(11):1171-82

12. van Es N, Coppens M, Schulman S, Middeldorp S, Büller HR. Direct oral anticoagulants compared with vitamin $\mathrm{K}$ antagonists for acute symptomatic venous thromboembolism: evidence from phase 3 trials. Blood 2014; 18; 124(12):1968-75

13. Vílchez JA, Gallego P, Lip G, Safety of new oral anticoagulant drugs: a perspective , Ther Adv Drug Saf. 2014; 5(1): 8-20 
14. Agnelli G, Becattini C, Franco L., New oral anticoagulants for the treatment of venous thromboembolism. Best Pract Res Clin Haematol. 2013; 26(2):151-61

15. Trkulja V., Safety of apixaban for venous thromboembolism prophylaxis: the evidence to date. Drug Healthc Patient Saf. 2016; 18; 8:25-38

16. Toth PP, Considerations for long-term anticoagulant therapy in patients with venous thromboembolism in the novel oral anticoagulant era. Vasc Health Risk Manag. 2016; 10; 12:23-34

17. HAS-BLED Score for Major Bleeding Risk, Available from: http://www.mdcalc.com/has-bled-score-for-major-bleeding-risk/

18. Hijazi Z, Oldgren J, Lindbäck J, Alexander J, Connolly S, Eikelboom J, et al, The novel biomarker-based ABC (age, biomarkers, clinical history)-bleeding risk score for patients with atrial fibrillation: a derivation and validation study, Lancet, 387, No. 10035, p2302-2311, June 2016 DOI: http://dx.doi. org/10.1016/S0140-6736(16)00741-8

19. Liew A, Eikelboom JW, O'Donnell M, Hart RG., Assessment of anticoagulation intensity and management of bleeding with old and new oral anticoagulants. Can J Cardiol. 2013; 29(7 Suppl): S34-44

20. European Medicines Agency (latest renewal Jan. 2016) Available from: <http://www.ema.europa.eu>

Publish in International Archives of Medicine

International Archives of Medicine is an open access journal publishing articles encompassing all aspects of medical science and clinical practice. IAM is considered a megajournal with independent sections on all areas of medicine. IAM is a really international journal with authors and board members from all around the world. The journal is widely indexed and classified Q1 in category Medicine. 